Title:

\title{
Erzählen im arabischen adab. Zwischen Fiktionalität und
} Faktualität.

Author(s):

Toral-Niehoff, Isabel

Document type: Preprint

Terms of Use: $\quad$ Copyright applies. A non-exclusive, non-transferable and limited right to use is granted. This document is intended solely for personal, non-commercial use.

Citation:

"Toral-Niehoff, Isabel. (2018). „Erzählen im arabischen adab. Zwischen Fiktionalität und Faktualität. Geschichte der Fiktionalität. Herausgegeben von Dr. Johannes Franzen, Patrick Galke-Janzen, Frauke Janzen, Marc Wurich. Ergon Verlag. S. 117-132. https://refubium.fuberlin.de/handle/fub188/33250 (Refubium)" 


\title{
Erzählen im arabischen adab. Zwischen Fiktionalität und Faktualität ${ }^{1}$
}

\author{
Isabel Toral-Niehoff
}

\section{Einfübrung}

$\mathrm{Zu}$ den besonderen Herausforderungen für die Erforschung von Fiktionalität in der Vormoderne gehört die Existenz von hybriden Textformen. Mittelalterliche Chroniken und Hagiographien entziehen sich z.B. einer Klassifizierung in die alternativen Kategorien Geschichte (faktual) oder Literatur (fiktional); zudem verweisen sie auf ein besonderes Verständnis von poetischer Wahrheit, was zu mancher Irritation geführt hat. Innerhalb der Mediävistik bewegt sich die Fiktionialitätsdebatte deshalb dahingehend, alternative Fiktionskonzepte zu entwerfen. ${ }^{2}$

Das Problem der hybriden Textformen stellt sich in ähnlicher Weise in der mittelalterlichen hochsprachlichen arabischen Literatur des 8.-10. Jahrhunderts (im Folgenden: MHAL), für die Stefan Leder feststellte:

The existence of fictive elements [...] cannot seriously be rejected [...] fictional narration, wherever it appears, is embedded in a mainstream of factual, or allegedly factual, narration [...]. This ambiguity often obstructs any attempt to decide which text, or which part of a text should be regarded as fiction. ${ }^{3}$

Leder setzte sich deshalb in seinem grundlegenden Artikel zu der Frage das Ziel, „to unmask the fictive narrative and thus prevent fiction from declaring itself a reality “4, was er als seine besondere Gefahr in der MHAL betrachtete. Schließlich gilt laut der communis opinio die MHAL als grundsätzlich ablehnend gegenüber Fiktion, so dass Fiktionalität demnach nur „maskiert“ oder in der Trivialliteratur auftreten könne. ${ }^{5}$

1 Dieser Artikel wurde im Rahmen des Courant-Forschungszentrum "Bildung und Religion" (Universität Göttingen) verfasst, das von der deutschen Exzellenzinitiative finanziert wird, und mit der Unterstützung eines Forschungsprojekts unter der Leitung von Dr. Delfina Serrano, finanziert durch das spanische Ministerium für Forschung und Innovation (FFI2013-43172-P). Ferner möchte ich hiermit Jens Scheiner (Göttingen), Undine Ott (Göttingen) und Yoones Dehghani Farsani (Göttingen) für ihre wertvollen Vorschläge und Korrekturen herzlich danken.

2 Vgl. den Überblick zur Debatte in der Mediävistik in Jan-Dirk Müller (2004) „Literarische und andere Spiele. Zum Fiktionalitätsproblem in vormoderner Literatur." Poetica 36. S. 281-312.

3 Stefan Leder (1998) „Conventions of Fictional Narration in Learned Literature”. Storytelling in the framework of non-fictional Arabic literature. Hg. Stefan Leder. Wiesbaden: Harrassowitz. S. 34-60, hier S. 34.

4 Leder (1998). S. 46.

5 Für einen Überblick über die Debatte der Wertigkeit von Fiktion in der MHAL und deren „Verbannung“ in triviale Genres s. Isabel Toral-Niehoff (2015a) „Fact and Fiction“ in der 
Inwiefern kann in der MHAL aber tatsächlich von einer „Einbettung“ fiktionaler Erzählelemente in einem faktualen Kontext gesprochen werden? Ist es möglich, diese Elemente mit Hilfe von Fiktionalitätssignalen zu isolieren? Wie können wir die oben genannte ,ambiguity“ zwischen Faktualität und Fiktionalität in der MHAL näher beschreiben?

Da die Kategorien „Fiktionalität“ und „Faktualität“ auf der Grundlage europäischer Texte aus der Moderne entwickelt wurden, dürfte es hilfreich sein, diese auch im Bereich der MHAL anzuwenden, um die Grenzen von deren Gültigkeit weiter auszuloten. Dies soll im Folgenden an zwei ausgewählten Beispielen des mittelalterlichen arabischen adab erfolgen, da diese Textgruppe am ehesten unserem modernen Verständnis von „Literatur" entspricht, insofern er neben der Dichtung den typischen Bereich ästhetischer Rede in der MHAL bezeichnet. ${ }^{6}$ Deshalb besteht auch im Rahmen von adab am ehesten die Möglichkeit, dass wir fiktionales Erzählen antreffen. ${ }^{7}$

Im Vorfeld sind allerdings einige Präzisierungen zu den Differenzen zwischen $a d a b$ und „Literatur“ notwendig, da wir es mit zwei Begriffen aus verschiedenen Kultur- und Zeitkontexten zu tun haben, und sie deshalb nur bedingt deckungsgleich sind. In den gängigen europäischen Literaturgeschichten der MHAL wird unter der Rubrik adab bzw. adab-Literatur eine breite Textgruppe geführt, die als „belles-lettres“ oder „schöngeistige Literatur“ bezeichnet und von anderen Kategorien wie Geschichtsschreibung und religiöser Literatur abgegrenzt wird. ${ }^{8}$ Viele der adab-Werke haben die Form großer thematisch geordneter Kompilationen

mittelalterlichen arabischen Literatur. Anmerkungen zu einer Debatte“. Faktuales und fiktionales Erzäblen. Interdisziplinäre Perspektiven. Hgg. Monika Fludernik, Nicole Falkenhayner, Julia Steiner. Würzburg: Ergon. S. 59-75, mit weiterführender Literatur.

6 Im heutigen modernen Standardarabisch bedeutet $a d a b$ „Literatur“ im modernen, europäischen Sinne. Dies ist allerdings eine spätere Bedeutungsentwicklung. $\mathrm{Zu}$ einer näheren Begriffsbestimmung von adab s.u. Zu Poesie als Bereich der Fiktion in der MHAL s. den Überblick bei Philipp Kennedy (2005) „Preface“. On Fiction and Adab in Medieval Arabic Literature. Hg. Philip Kennedy. Leiden: Brill. xi-xxii.

7 Die Definition von „Literatur“ ist theorieabhängig und kann in diesem Rahmen unmöglich reproduziert werden, hier sei auf den Überblick bei Burckhard Menninghof (2007) „Literatur“. Metzler Lexikon Literatur. Hgg. Dieter Burdorf/Christoph Fasbender/Burckhard Moennighoff. Stuttgart: Metzler. S. 445a-445b sowie Lothar van Laak (2007) "Fiktionalität”. Metzler Lexikon Literatur. Hgg. Dieter Burdorf/Christoph Fasbender/Burckhard Moennighoff. S. 240a?-242a verwiesen. Demnach wird „Literatur“ essentialistischen Definitionen zufolge als der konventionelle Rahmen für Fiktion gesehen und darüber definiert; „Literatur“ sei also durch diese für sie spezifische Art des Behauptens und Fingierens („als-ob“) gekennzeichnet; antiessentialistische Konzepte sehen hingegen „Literatur“ eher als Institution, andere betonen den Aspekt der Rezeption.

8 S. z.B. Julia Ashtiany u.a., Hgg. (1990). The Cambridge History of Arabic Literature. 'Abbasid Belles-Lettres. Cambridge: Cambridge University Press; M. J. L. Young/J. D. Latham/R. B. Serjeant, Hgg. (1990) The Cambridge History of Arabic Literature. Religion, learning and science in the 'Abbasid period. Cambridge: Cambridge University Press. Auf das Problem der Konfusion zwischen etischen und emischen Kategorien weist Hoyland hin: Robert G. Hoyland (2006) „History, Fiction and authorship in the first centuries of Islam“. Writing and Representation in Medieval Islam. Hg. Julia Bray. Routledge: London. S. 16-46, hier S. 18. 
von einzelnen Episoden, Anekdoten, Sentenzen und Gedichten, in denen Rhetorik, Kunstsprache und Humor eine große Bedeutung besitzt. In der hier erkennbaren ästhetischen Zielsetzung von adab deckt es sich mit „Literatur" im konventionellen, europäischen Gebrauch. Zudem wird aber adab-Werken auch eine didaktische Intention zugeschrieben, was sie wiederum in die Nähe von Sachtexten rückt. ${ }^{9}$ Ähnliches resultiert aus der engen thematischen Verwandtschaft von adab mit Weisheitsliteratur und Fürstenspiegeln. ${ }^{10}$

Die Semantik von adab geht zudem weit über die Bedeutungsdimension einer Textgruppe hinaus. So kann der schillernde Begriff auch „feine Sitte“, „urbanitas“, „Bildung“ wie auch „Fertigkeiten eines bestimmten Berufes“ bedeuten. Offenbar bezeichnet er sowohl die Beschäftigung mit Poesie, Sprache, Weisheitssprüchen und erhabener Rede als Zeichen sozialer Distinktion, wie auch die Texterzeugnisse selbst, hat also eine passive und aktive Bedeutung zugleich. In vielen Aspekten kommt adab somit dem griechischen enkyklios paideia nahe. ${ }^{11}$ Im Zentrum des adab steht das Ideal des adīb, des wahrhaft „Gebildeten“, der sich weniger durch die vertiefte Kenntnis bestimmter Wissensgebiete auszeichnet als durch die Breite seines Wissens, dies verbunden mit der Fähigkeit, dieses Wissen in gesellschaftlich angemessener Form anzuwenden. Adab-Werke stellen somit das Wissen zur Verfügung, das von einem adỉb erwartet wird.

Vor dem Hintergrund der genannten Schwierigkeiten in der Definition von adab und dem Mangel an Vorarbeiten kann im Folgenden folglich nur ein sehr vorläufiger Einblick gegeben werden.

$9 \mathrm{Zu}$ verschiedenen Versuchen, $a d a b$ zu definieren, s. Jaakko Hämeen-Anttila (2015) „Adab a) Arabic, early developments“. Encyclopaedia of Islam, THREE. Hgg. Kate Fleet u,a. Brill Online; Isabel Toral-Niehoff (2010) „,Sei seine Dienerin, dann wird er dein Diener sein! Auf der Suche nach der idealen Ehefrau: Ibn 'Abd Rabbihi und sein Buch über die Frauen“. Didaktisches Erzäblen. Spielarten literarischer Belehrung in Orient und Okzident. Hgg. Regula Forster/Romy Günthart. Frankfurt am Main: Peter Lang. S. 255-275, hier S. 257 (mit weiterführender Literatur); auch Hartmut Fähndrich (1990) „Der Begriff ,adab und sein literarischer Niederschlag“. Neues Handbuch für Literaturwissenschaft. Bd. 5. Orientalisches Mittelalter. Hg. Wolfhart Heinrichs. Wiesbaden: Aula. S. 326-345; Tarif Khalidi (1994) Arabic Historical Thought in the Classical Period. Cambridge: Cambridge University Press. S. 83-130.

$10 \mathrm{Zu}$ adab als „web of myth“" s. Julia Bray (2005) „'Abbasid Myth and the Human Act: Ibn 'Abd Rabbih and Others". On Fiction and Adab in Medieval Arabic Literature. Hg. Philip Kennedy. Leiden: Brill. S.1-54. Zur Nähe zwischen adab und Fürstenspiegeln s. Isabel Toral-Niehoff (2015b) „,The Book of the Pearl on the Ruler' in the Unique Necklace by Ibn 'Abdrabbih: Preliminary Remarks." New Approaches to the History of Political Thought: Mirrors of Princes Reconsidered. Hgg. Neguin Yavari/Regula Forster. Cambridge: Harvard University Press. S. 134-151.

11 Der Vergleich von adab mit paideia findet sich u.a. in Khalidi (1994). S. 83; eine weitere Ausarbeitung dieser Parallele steht allerdings noch aus. 


\section{Text 1: Aus dem „Einzigartigen Halsband“}

Der erste adab-Text stammt aus dem 'Iqd al-farìd („Das [einzigartige] Halsband“), einer enzyklopädischen Sammlung, die in al-Andalus, dem islamisch beherrschten Teil der iberischen Halbinsel, des 10. Jahrhunderts entstand. Der für die arabische Literatur typische bildhafte Buchtitel ist nicht nur Zierde, sondern auch Programm, denn der Verfasser wollte „eine Auswahl der besten aller Edelsteine“ aus dem $a d a b$ präsentieren, wie er in seiner Einführung selbst sagt: In seinem Werk sind demnach „verschiedene Edelsteine der Rede enthalten, aufgereiht auf feinen Fäden und wohl angeordnet“, d.h. es besteht aus 25 einzelnen Büchern oder Großkapiteln, die - jeweils mit dem Titel eines Edelsteins, jawehar, versehen - nach thematischen Gesichtspunkten systematisch erfasst sind. Alle diese Bücher ergeben zusammen eine „Kette“ bzw. ein „Halsband“. Im Folgenden wird ein Abschnitt vorgestellt, der aus Buch XIV stammt, das sich monographisch mit der Kalifengeschichte beschäftigt. ${ }^{12}$

Der erste umayyadische Herrscher in al-Andalus war 'Abd al-Rahmān b. Mu'āwiya, er übernahm die Herrschaft am Freitag, des letzten Zehntels des Dhūl-Hijja des Jahres 138, da war er 28 Jahre alt. Er starb am 10. des ersten Jumāda im Jahr 172. Seine Herrschaft dauerte 32 Jahre und 5 Monate. ${ }^{13}$

Man nannte ihn „den Falken der Quraysh“. ${ }^{14}$ Das war nämlich so: Eines Tages fragte der Kalif al-Manșūr einige seiner Begleiter: „Wer ist der Falke der Quraysh?“ Sie antworteten: „Der Befehlshaber der Gläubigen (al-Manșūr selbst), denn er befestigte ein Reich, bezwang dort die Unruhen und befreite (das Reich) von Missständen.“ „Nein, Ihr habt unrecht.“ „Dann etwa Mu'āwiya? „ „Nein.“ „'Abd al-Malik b. Marwān?“ „Nein.“ „Dann wer, o Befehlshaber der Gläubigen?“ Er sagte: „Der Falke der Quraysh ist 'Abd al-Rahman, der das Meer durchquerte, Armut ertrug, alleine ein barbarisches Land betrat, dort Städte gründete, ein Heer aufstellte, die Verwaltung aufbaute, ein Königreich aufbaute, nachdem es sich abgetrennt hatte, mit Hilfe seiner trefflichen Führerschaft und seinem starken Willen. Mu'āwiya bestieg ein Pferd, das schon zuvor für ihn von 'Umar und 'Uthmān gesattelt worden war; 'Abd al-Malik, weil er zuvor dafür ernannt worden war, und der Befehlshaber der Gläubigen (al-Manșūr selbst) [bestieg das Pferd] dank des Kampfes seiner Angehörigen und die Solidarität seiner Anhänger. Aber 'Abd al-Rahmmān tat dies alleine, einzig mit der Unterstützung seiner Urteilskraft, und begleitet von seinem starken Willen. “15

Für die Interpretation dieser Anekdote ist aufgrund ihrer politischen Implikationen der historische Entstehungskontext von besonderer Bedeutung. Der Verfas-

12 Zu diesem Buch s. Isabel Toral-Niehoff (2015c) „History in Adab Context: ,The Book on Caliphal Histories' by Ibn 'Abd Rabbih (246/860-328/940)". Journal of Abbasid Studies 2. S. 61-85. hte also 756-789.

Quraysh ist der Name eines angesehenen mekkanischer Stammesverbandes, der zur Zeit des Muhammad die Hegemonie hatte. Zu ihm gehörte der Prophet Muhammad, die Umayyaden und auch die 'Abbasiden. Laut sunnitischer Lehre sollte der Kalif ein Mitglied der Quraysh sein.

Ibn 'Abd Rabbih (1990) al-'Iqd al-farìd. Hg. Aminn/al-Abyārī/Tudmirī. Beirut: Dar al-Kutub al-IlmiyaBd. IV S. 443. 
ser/Kompilator Abū 'Umar Ahmad b. Muhammad b. 'Abdrabbih al-Andalusi (860-940) stammte aus der damaligen Kalifen-Residenzstadt Cordoba und stand über ein Klientelverhältnis der regierenden Umayyadendynastie sehr nahe, womöglich war er eine Art Hofpanegyriker. ${ }^{16}$ Im Jahr 929 - während der vermuteten Kompilationszeit des „Halsbandes“ - proklamierte sich der regierende Umayyadenemir 'Abd al-Rahmmān (reg. 912-961) zum Kalifen und gründete so das Kalifat von Cordoba. Die Titelübernahme symbolisierte das Hegemoniestreben der umayyadischen Dynastie innerhalb der damaligen islamischen Welt, denn die Errichtung eines Kalifats bedeutet aus islamischer Sicht einen universellen Herrschaftsanspruch. ${ }^{17}$ So provozierte die Erklärung die in Bagdad regierenden Abbasiden ${ }^{18}$, denn schließlich hatten Letztere im Jahr 750 die damals regierende Umayyadendynastie in Damaskus in einer „Revolution“ gewaltsam abgelöst. Die Umayyaden in al-Andalus waren deren Nachkommen und leiteten ihre Legitimität von dieser Genealogie ab. Kurzum, die Rivalität mit der Dynastie der Abbasiden in Bagdad bestimmte entscheidend das Selbstverständnis der Umayyaden in al-Andalus. ${ }^{19}$

Die hier zitierte Anekdote befindet sich im Buch XIV, das sich monographisch der Kalifengeschichte widmet. Aufgrund des besonderen regionalpolitischen Kontextes folgt die Kalifengeschichte des „Halsbandes“ allerdings einer „westlichen“, pro-umayyadischen Perspektive und setzt die Linie der legitimen Kalifen nach 750 mit den umayyadischen Emiren aus al-Andalus fort, unter Auslassung der Abbasiden, die somit zu Usurpatoren degradiert werden. ${ }^{20}$ Diese Geschichte leitet die Sektion über die Umayyaden in al-Andalus ein und befasst sich mit dem Gründer der Umayyadenherrschaft in Spanien, dem Prinzen 'Abd al-Raḩmān I. (reg. 756-789). Dieser war derjenige Abkömmling der abgesetzten Umayyaden aus Damaskus, der im Jahr 756 auf der iberischen Halbinsel die Macht übernommen und dann das umayyadische Emirat von al-Andalus gegründet hatte. Seine abenteuerliche Flucht vor dem grausamen Massaker der Abbasiden an seinen Verwandten in Syrien und seine Machtübernahme auf der Halbinsel wurden zum Gründungsmythos der Dynastie in al-Andalus. ${ }^{21}$

Die namentlich genannten Personen dieser Erzählung sind somit alle dem Leser wohlbekannte historische Figuren aus dem Tableau der frühen islamischen Geschichte. So handelt es sich bei al-Manșūr um den bedeutenden frühen Abba-

16 Zu seiner Biographie S. Walter Werkmeister (1983) Quellenuntersuchungen zum Kitäb al-`Iqd al-Farid des Andalusiers Ibn' Abdrabbih (246/840-328/940). Berlin: Schwarz. S. 16-26.

$17 \mathrm{Zu}$ einer allgemeinen Bewertung der Kalifenideologie in al-Andalus mit weiterführender Literatur, s. Janina Safran (2000) The Second Umayyad Caliphate: The Articulation of Caliphal Legitimacy in al-Andalus. Cambridge: Harvard University Center for Middle Eastern Studies.

18 Vermutlich bildete allerdings die Proklamierung des Kalifats der Fatimiden in Nordafrika den unmittelbaren Anlass (909): s. Safran (2000), Index s.v. Fatimids.

19 S. Safran (2000). S. 120 und Index, s.v. Abbasids; Toral-Niehoff (2015c) S. 71-73.

20 Für diese Liste s. Toral-Niehoff (2015c) S. 67-71.

21 Safran (2000). S. 120; Toral-Niehoff (2015c). S. 71-73. Siehe auch Sabatino Moscati (1950) „Le massacre des Umayyades“. Archiv orientální 28, 4. S. 88-115. 
sidenkalifen (reg. 754-775); ${ }^{22} \mathrm{Mu}$ 'āwiya (reg. 661-680) ist wiederum der Gründer der Umayyadenherrschaft in Syrien;23 'Abd al-Malik (reg. 685-705) war der bedeutendste der Umayyadenkalifen; ${ }^{24}$ und 'Abd al-Rahmmān (reg. 756-789) ist der Name des Umayyadenprinzen, der in al-Andalus das Emirat gründete. Gemeinsam ist Mu'āwiya, 'Abd al-Malik und 'Abd al-Rahmān, dass sie jeweils die Herrschaft der Umayyaden nach einer Periode der Instabilität festigten.

Die Anekdote hat eine intensive politische Resonanz, erzählt sie doch, wie ausgerechnet der Erzfeind der Umayyaden, der Abbasidenkalif al-Manșūr, seine Bewunderung für den Umayyadenprinzen ausdrückt. Zusätzlich etabliert sie eine Typologie von vorbildhaften Umayyadenherrschern, die erfolgreich gegen politisches Chaos kämpften, zu denen schließlich implizit der regierende Kalif 'Abd alRaḥmān III. gerechnet wird, der nicht nur genealogisch, sondern auch über seine Namensgleichheit mit dem hier gelobten Prinzen 'Abd al-Rahmmān verbunden war. Die Geschichte kontrastiert zudem mit dem verbreiteten Bild des al-Mansūu in den arabischen Quellen: In Texten aus al-Andalus repräsentiert er den frühen Abbasidenkalifen, der die Umayyaden gnadenlos in Syrien verfolgte; aber irakische Texte betonen seine anti-umayyadische Haltung. ${ }^{25} \mathrm{Da}$ diese Anekdote nur im Westen der islamischen Welt zirkulierte ${ }^{26}$, handelt es sich somit höchstwahrscheinlich um eine historische Konstruktion, die dazu dienen sollte, den besonderen Status des Umayyadenprinzen 'Abd al-Rahmmān zu unterstreichen, dem sogar sein Erzfeind aufgrund von dessen Heldentaten Lob zollen „musste“. In diesem Sinne dürfen wir also von einer typischen historischen Fiktion ausgehen, die für politische und legitimatorische Ziele funktionalisiert wurde. Dennoch bleibt die Erzählung hybride.

Für einen faktualen Status spricht nicht nur die Historizität der Personen, sondern auch gerade die politische Funktionalisierung der Anekdote, deren Bedeutung ja davon abhängt, dass dieser Dialog bzw. die Anerkennung durch alManșūr tatsächlich stattgefunden hat. Die externe Fokalisierung und der realistische Stil der Geschichte evozieren zudem Wirklichkeitsnähe und weisen somit auf einen faktualen Status hin.

Es überwiegen allerdings die Hinweise auf einen fiktionalen Status. Dafür spricht zunächst die starke Literarisierung und Dramatisierung der Anekdote. So

22 Al-Manșūr war der zweite der Abbasidenkalifen und gilt als der eigentliche Begründer des abbasidischen Reiches, er war u. a. Gründer der Stadt Bagdad (762).

23 Mu'āwiya b. Abi Sufyān übernahm die Herrschaft nach den Wirren unter dem Kalifat von 'Ali (656-661) und der Schlacht von Șiffin (657). Er verlegte die Hauptstadt des jungen Kalifats von Medina nach Damaskus und gilt als Begründer der Umavyadenherrschaft.

'Abd al-Malik b. Marwān musste die ersten Jahre seines Kalifats der Sicherung der Herrschaft der Umayyaden in Syrien, Palästina und Ägypten widmen. Zu den Schwierigkeiten, mit denen er zu kämpfen hatte, zählten mehrere verheerende Pestepidemien, Hungersnöte und byzantinische Angriffe auf Syrien, so wie ein Gegenkalifat auf der Arabischen Halbinsel. Mit ihm beginnt die Herrschaft des marwanidischen Zweigs der Umayyadendynastie, zu dem auch die Umayyaden in al-Andalus gehörten.

25 Toral-Niehoff (2015c). S. 73.

$26 \mathrm{Zu}$ weiteren Versionen s. Toral-Niehoff (2015c). S. 72, Anm. 53. 
hat der erste Teil die Form eines Rätseldialogs, welcher einem dramatischen Höhepunkt zustrebt, nämlich der Lösung des Rätsels. Der zweite Teil, in dem alManșūr sein hochrhetorisches Lob über 'Abd al-Rahmmān anstimmt, ist nicht nur durchsetzt von Hyperbeln und Metaphern, die in einem sehr raschen Tempo aneinandergereiht werden, sondern folgt auch dem stark rhythmischen, parallelistischen Stil mit gelegentlichem Binnenreim, der die erhabene Prosa im Arabischen auszeichnet. ${ }^{27}$ Hier scheinen ästhetische Gesichtspunkte und die „tellability" der Geschichte für die Auswahl eine bedeutende Rolle gespielt zu haben.

Ein anderer wichtiger Hinweis auf Fiktionalität resultiert aus der Tatsache, dass Ibn 'Abd Rabbih dieser Anekdote keinen isnād (Autoritätenkette) beifügt. Der isnād ist in seinem Originalkontext eine Authentifizierungsstrategie aus den religiösen Wissenschaften, wo er die kanonische Gültigkeit des badith (prophetischer Traditionen) bestimmen sollte. ${ }^{28}$ Der isnād hat sich ab dem 9. Jahrhundert auch allmählich in anderen Bereichen der MHAL ausgebreitet, so wie z.B. in der Geschichtsschreibung ${ }^{29}$ und gelegentlich auch in der adab-Literatur, wie wir im zweiten Text sehen werden. Die Nicht-Verwendung eines isnād im „Halsband“ wirft ein interessantes Licht auf dessen Status. Ibn 'Abd Rabbih begründet seine Omission des isnād am Anfang seines Gesamtwerkes und führt ausdrücklich ästhetische Erwägungen an. So macht er in seiner generellen Einführung einerseits geltend, dass er den isnād für das flüssige Lesen als störend empfindet; andererseits betont er, dass er ohnehin Nachrichten überliefere wie „Geschichten, Weisheitssprüche und Anekdoten, denen der isnād in seiner Lückenlosigkeit weder nützt, wie ihm sein Fehlen schadet." ${ }^{\text {30 }}$ Die Positionierung von Ibn 'Abd Rabbih verdeutlicht, dass seine Auslassung eine bewusste Entscheidung war, und dass er eine gute Kenntnis von dem isnād als Kulturtechnik besaß, umso mehr als seine Lehrer zu den Gelehrten gehörten, denen die Einführung der ḅadith - Wissenschaft und damit des formalisierten isnād in Al-Andalus zugeschrieben wird. ${ }^{31}$ Seine Aussage zeigt auch, dass er die Gültigkeit seiner Zitate nicht von ihrer Au-

27 Hierzu s. A. F. Beeston (1983) „The role of parallelism in Arabic prose“. The Cambridge History of Arabic Literature. Arabic Literature to the end of the Umayyad period. S. 180-185; Stefan Leder/Hilary Kilpatrick (1992) „Classical Arabic Prose Literature. A Researcher's Sketch Map“. Journal of Arabic Literature 23, 1. S. 2-26.

28 Für einen guten Überblick zur Technik des isnād und der binnenislamischen badith-Kritik vgl. Jonathan A. C. Brown (2009) Hadith. Oxford: OneWorld. S. 67-122; ferner Übersichtsartikel von Jens Scheiner (2016, S. 110- 131,Der Hadit“, in: Islam. Hg. Rainer Brunner. Stuttgart: Kohlhammer.

29 Zur Ausbreitung der Technik des isnād in der Geschichtsschreibung vgl. Khalidi (1994). S. 17-81; für die Verwendung in der adab-Literatur vgl. Khalidi (1994). S. 99-100; s. auch Werkmeister (1983). S. 44-46 zu seiner Auslassung im „Halsband“. Die Funktion und Verwendung des isnād außerhalb des hadith bedarf aber m.E. noch einer vertieften Untersuchung. Siehe auch unten zum isnād im „Buch der Lieder“.

30 Werkmeister (1983). S. 44.

31 Nämlich die beiden Gelehrten Baqi b. Makhlad (gest. 889) und Muhammad b. Waḍdāh (gest. 900). Zu ihnen siehe Isabel Fierro (1989) „The introduction of hadith in al-Andalus“. Der Islam 66, 1. S. 68-93. 
thentifizierung und damit auch von ihrer Faktizität abhängig machte; sie sind somit ohne Kontext universell gültig.

Auf keinen Fall handelt es sich hier um einen Fall autonomer Fiktionalität, da diese keineswegs „entblößt" wird, sondern gegebenenfalls um eine funktionale Fiktionalität, die vorhandenes Material rhetorisch aufbereitet. ${ }^{32}$ Dennoch fällt es schwer zu entscheiden, welcher narratologische Status dieser Geschichte in ihrem Originalkontext zukam. Auffallend ist schließlich auch der Kontrast zwischen dem realistischen Stil der Rahmenerzählung, die Faktizität evoziert, und dem rhetorischen Stil der wörtlichen Rede, der eine Fiktionalisierung signalisiert.

\section{Text 2: Aus dem „Buch der Lieder“}

Der folgende Text stammt aus dem Sammelwerk „Das Buch der Lieder“ des Abū 1-Farağ al-Ișfahānī (897-967). Das umfangreiche $\mathrm{Werk}^{33}$ ist eine Sammlung von über 100 Liedern bzw. Liedtexten; zudem enthält sie biographische Notizen zu den jeweiligen Sängern, Dichtern und Musikern, die sich gelegentlich zu umfangreichen historischen Exkursen auswachsen. ${ }^{34}$ Wegen dieses reichen historischen Materials, das zudem in einer eleganten und gut lesbaren Erzählprosa geschrieben ist, werden Auszüge aus dem „Buch der Lieder“ gerne zitiert und übersetzt. ${ }^{35}$ Es wird, so wie andere literarische Anthologien, der schon genannten umfangreichen Kategorie der adab-Texte zugeordnet, ${ }^{36}$ denn „,compiling literary anthologies was a central activity for the cultivation of $a d a b .{ }^{\text {"37 }}$

Im Unterschied zu dem zuvor genannten „Halsband“ finden wir im „Buch der Lieder" regelmäßig einen isnād (Überlieferkette) zu Beginn der jeweiligen Nachricht/Texteinheit. Der Verfasser verwendet somit die oben genannte Authentifizierungstechnik der Gelehrten in der prophetischen Tradition (badit), die sich auch in der Historiographie ausgebreitet hatte ${ }^{38}$, und die Ibn 'Abd Rabbih ausdrücklich ausgelassen hat. Diese Praxis hängt mit der expliziten Intention des

32 Müller (2004). S.282-283; siehe van Laak (2007). S. 240.

33 Das Werk umfasst in der Standardedition aus Kairo 26 Bände: al-Ișfāhānī (1927-1961)

Kitāb al-Ag̀̄añi. Kairo: Dār al-Kutub. $\mathrm{Zu}$ den verschiedenen Editionen und Teilübersetzungen vgl. Hilary Kilpatrick (2003) Making the Great Book of Songs. London: Routledge. S. 1-12.

34 Kilpatrick betont, dass das „Buch der Lieder“ vor allem als Liedersammlung zu lesen sei: Kilpatrick (2003). S. 13-23.

35 Kilpatrick (2003). S. 2 betont die „readability“ des Buches.

36 Fähndrich zählt z.B. das „Buch der Lieder“ zu den „adabisierten“ Werken, Fähndrich (1990). S. 339. Bilal Orfali (2012) „A Sketch Map of Arabic Poetry Anthologies up to the Fall of Baghdad“. Journal of Arabic Literature 43. S. 29-69 betont mehrfach den engen Zusammenhang zwischen Anthologien und $a d a b$ :,Literary anthology represents a type of $a d a b "$, S. 31.

37 Orfali (2012). S. 29.

38 Zum isnād s. Anm. 29; zur Verwendung des isnād im „Buch der Lieder“, s. Kilpatrick (2003). S. 94-104; siehe auch unten Anm. 40. 
Verfassers al-Iṣfahānī zusammen, die jeweiligen Lieder nicht nur zu sammeln, sondern auch durch Hinzufügung historischer und biographischer Notizen zu kontextualisieren; ${ }^{39}$ der isnād soll folglich diese Notizen durch eine Nennung von Autoritäten authentifizieren. Damit geht der Verfasser des „Buches der Lieder" ähnlich wie viele Verfasser literarischer Anthologien seiner Zeit vor, die einen isnād einfügten. ${ }^{40}$ Wie viele dieser Anthologen, erlaubt sich allerdings alIșfahāni einen flexibleren Umgang mit dem isnād als es die strenge Handhabung in seinem Originalkontext der Prophetentradition vorschrieb; ${ }^{41}$ die genaue Funktion dieser langen Namensketten im „Buch der Lieder“ ist jedoch unerforscht. Sie geht vermutlich über die einer reinen Authentifizierung der Nachrichten hinaus und diente auch literarischen Zielen. ${ }^{42}$ Im Folgenden werde ich diesen isnād jeweils nur in sehr verkürzter Form nennen, es genügt in diesem Rahmen der Hinweis auf dessen Position im Text und eine kurze Evaluation. ${ }^{43}$

ISNĀD (Diese Kette reicht durchgehend von dem Zeithorizont des Verfassers alIṣfahāni bis zu dem der erzählten Zeit, also das Jahr 17 H./638 CE; damit ist die Kette korrekt und valide)

Mughira ${ }^{44}$ pflegte eine Frau der Thaqif ${ }^{45}$ regelmäßig zu besuchen, die al-Raqtä ${ }^{-36}$ hieß Da traf ihn Abū Bakra ${ }^{47}$ und fragte ihn: „Wo gehst du hin?“ Er sagte: „Ich besuche die

literarischen Verwendung des isnād vgl. Kilpatrick (2003). S. 101: „he uses isnad to literary effect. [...] Finally, the isnad, the hallmark of serious scholarship, maybe combined with frivolous material, and this incongruous juxtaposition gives a tinge of irony to a passage."

43 Die Nennung der einzelnen Überlieferer würde zu weit führen; und auch das Problem der umstrittenen Echtheit dieser isnāde möchte ich an dieser Stelle nicht diskutieren, da es für diese Fragestellung irrelevant ist. Wichtig ist vielmehr, ob die jeweiligen isnäde den formaltechnischen Erfordernissen dieser etablierten Strategie genügten und somit als echt wahrgenommen werden konnten. Ich möchte mich hier ganz besonders bei Jens Scheiner für seine Erläuterungen bedanken. Medina früh anschloss. Er stammte aus der Stadt TTā'if südlich von Mekka und zeichnete sich als besonders geschickter und schlauer Politiker aus. Unter dem Kalifen 'Umar (reg. 634-644) wurde er Gouverneur von Bașra und dann aufgrund der hier erzählten Episode 638 abgesetzt; später wurde er mehrfach Gouverneur von Kūfa, unter anderem unter Kalif Mu`āwiya. Henri Lammens (2015) „al-Mughïra b. Shưba.” Encyclopaedia of Islam, Second Edition. Hgg.. P. Bearman u.a. Brill Online.

45 Thaqif ist der Name des Stammes, der die Stadt Țā̄if beherrschte. Zu ihm gehörte auch Mughira. Sie waren wichtige Verbündete der Quraysh (Vgl. Anm. 14), welche Mekka dominierten. Michael Lecker (2015) "Thakif". Encyclopaedia of Islam. Second Edition. Hgg. P. Bearman u.a. Brill Online.

46 Diese Frau ist ansonsten unbekannt.

47 Weniger bekannter Prophetengefährte, ehemaliger Sklave der Thaqif aus Abyssinien, der 630 vom Propheten freigelassen wurde, und der sich später in Bașra niederließ, wo er viele 
Familie von dem und dem.“ Da ergriff er ihn beim Kragen und rief: „Ein Emir wird besucht und besucht nicht selbst! ${ }^{\star 48}$

ISNĀD (Bei dieser Kette handelt es sich um einen komplexeren isnād, bei dem sich der letzte der gemeinsamen Überlieferer der jüngeren Kette jeweils auf mehrere ältere Überlieferungsstränge bezieht. Diesem hängt al-Iṣfahānī jeweils auch die einzelnen angefügten Ketten an, er geht also ungewöhnlich detailliert und gewissenhaft vor. Auch dieser lange isna $\bar{a}$, der in der Druckfassung ca. 2/3 der Seite umfasst, ist korrekt und valide) Mughira (b. Shu'ba) pflegte am helllichten Tag das Dār al-Imāra (Statthalterpalast) zu verlassen. Da traf ihn regelmäßig Abū Bakra, um ihn dann zu fragen: „Wo gehst du hin, o Emir?“ Er sagte dann: „Ich gehe, um eine Sache zu erledigen.“ Er fragte dann immer: „Was für eine Angelegenheit? Der Emir wird besucht und besucht nicht selbst!“

Und es war so, dass die Frau, die er besuchte, eine Nachbarin des Abū Bakra war. Als Abū Bakra eines Tages in einem seiner Zimmer mit seinen Freunden und seinen beiden Brüdern Nāfic und Ziyād ${ }^{49}$, sowie mit einem anderen Mann namens Shibl b. Ma'bad zusammensaß - wobei das Zimmer sich genau gegenüber dem Zimmer seiner Nachbarin befand - da schlug ein Windstoß die Tür der Frau auf und öffnete sie. Da schauten die Männer und, siehe da! Dort war Mughira und befand sich gerade mitten im Liebespiel mit ihr. Da sagte Abū Bakra: „Was für ein Verderben, in das wir hier geraten sind! Schaut hin!“ Da schauten sie, bis sie sicher waren ${ }^{50}$. Dann kam Abū Bakra hinab und setzte sich (vor das Haus?) hin, bis Mughira zu ihm hinauskam, nämlich aus dem Haus der Frau, und sagte dann zu ihm (Mughira): „Du weißt sehr wohl, wie die Sache steht, trete also von deinem Amt (für uns) zurück!“ Da ging Mughira los, um mit den Leuten das Mittagsgebet zu beten, da hinderte ihn Abū Bakra daran ${ }^{51}$ und sagte: „Bei Allah, du wirst nicht für uns vorbeten, nachdem du das getan hast, was du getan hast!" Die Leute aber sagten: „Lasst ihn, er soll für uns beten, er ist der Emir!“ „Und schreibt darüber (lieber) einen Brief an (den Kalifen) 'Umar!" Da schrieben sie ihm. Und sein (Antwortbrief) besagte, dass sie alle vor ihn zu bringen seien, Mughira und die Zeugen.

Diese Passage befindet sich in der umfangreichen Biographie des Prophetengefährten Mughira b. Shu'ba, die im „Buch der Lieder“ aufgenommen wurde. ${ }^{52}$

Nachkommen hinterließ. M. Th. Houtsma/Ch. Pellat (2015) „Abū Bakra“. Encyclopaedia of Islam. Second Edition. Hgg. P. Bearman u.a. Brill Online.

48 Diese Formel wiederholt sich auch weiter unten.

49 Abū Bakra, Nāfi` und Ziyād waren Halbbrüder. Ziyād b. Abihi war zudem eine bekannte politische Persönlichkeit und wurde später Gouverneur des Irak. S. I. Hasson (2015) „Ziyād b. Abihi“. Encyclopaedia of Islam. Second Edition. Hgg. P. Bearman u.a. Brill Online.

50 Der Tatbestand der Unzucht (zina $\left.\vec{a}^{\top}\right)$ erfordert nach islamischem Recht vier männliche Augenzeugen des Geschehens, damit er als erwiesen gilt; können letztere das Zeugnis nicht in aller Genauigkeit erbringen („wie ein Stab in einer Kuhlflasche auf und ab geht“), werden diese Zeugen bestraft. R. Peters (2015) „Zinā'“. Encyclopaedia of Islam. Second Edition. Hgg. P. Bearman u.a. Brill Online. Wie wir später sehen werden, beruht die Pointe in dieser Geschichte genau auf dieser Regelung.

51 Das islamische Gebet erfordert einen Zustand der rituellen Reinheit, der durch Geschlechtsverkehr (erst recht durch Unzucht) verloren geht. Dass Mughira in diesem Zustand für die Gemeinde vorbeten wollte (seine Aufgabe als Gouverneur), ist somit ein zusätzlicher Skandal.

52 Die gesamte Biographie des Mughīra befindet sich in al-Ișfāhānī (1927-1961), Bd. XVI. S. 78-101; dieser Abschnitt auf S. 94-95. Eine Teilübersetzung ins Deutsche findet sich bei Gernot Rotter (2004) Und der Kalif beschenkte itn reichlich. Auszüge aus dem „Buch der Lieder“. Lenningen: Erdmann. S. 26-39. Die Episode der „Unzuchtsbezichtigung“ auf S. 36-39. 
Den Aufhänger für diese Biographie bildet die Tatsache, dass Mughïr, der sich sonst nicht als Dichter oder Musiker einen Namen gemacht hat, sondern als Politiker, den Text eines Liedes ${ }^{53}$ verfasst haben soll, das am Anfang des Kapitels zitiert wird. Vermutlich dürfte aber vor allem sowohl die Unterhaltsamkeit („tellability") der darin enthaltenen Anekdoten sowie die satirische und moralische Dimension seiner Vita eine große Rolle für die ausführliche Aufnahme in das „Buch der Lieder" gespielt haben, wie wir im Folgenden sehen werden. ${ }^{54}$

Die pikante Erzählung ist Teil einer längeren Episode, in der es um eine causa celebris geht, nämlich um den Prozess gegen Mughira wegen Unzucht im Jahre 17/638, als dieser Gouverneur von Bașra unter dem Kalifen 'Umar war. Sie erscheint auch in anderen Sammlungen (so z.B. im historischen Werk des alTTabari) mit einigen Varianten, die hier nicht im Einzelnen diskutiert werden sollen. ${ }^{55}$ Gemeinsam ist allen Berichten der Hauptplot, nämlich dass der amtierende Gouverneur Mughira von Abū Bakra der Unzucht bezichtigt wurde, was nach islamischem Recht mit dessen Hinrichtung bzw. Auspeitschung bestraft werden musste. ${ }^{56}$ Mughira wurde aber freigesprochen, weil sein Prozessgegner nicht alle vier der erforderlichen männlichen Augenzeugen aufweisen konnte. ${ }^{57}$ Die Diskussion der juristischen und historischen Implikationen dieses Prozesses soll hier nicht Thema sein, wichtig ist in diesem Rahmen vielmehr das Bild, dass alIșfahānì von Mughìra zeichnet, nämlich das eines Picaros, dessen Klugheit und Scharfsinn ihn aus den schwierigsten Lagen zu retten vermag. ${ }^{58}$ Diese romantisierende Charakterisierung prägt die Version des Prozesses im „Buch der Lieder“, welche sich fast wie eine spannende und witzige „Sex-and-Crime“-Geschichte liest. So impliziert al-Ișfahānī, dass Abū Bakra ohnehin gegen Mughïra voreingenommen war und nur darauf wartete, ihn bei einer unziemlichen Handlung zu erwischen; dann beschreibt der Verfasser detailliert wie Mughira zu allerhand Tricks, Manipulation und Erpressung griff, um Zeit zu gewinnen und einen der Zeugen zu überzeugen, nicht gegen ihn auszusagen. ${ }^{59}$ Die Aussagen der vier Zeugen im Prozess werden dann in einem wörtlichen Dialog hochdramatisch inszeniert ${ }^{60}$ und bringen schließlich die Wende, als der letzte Zeuge zwar bezeugt,

53 al-Iṣfāhānī (1927-1961). Bd. XVI. S. 78.

54 Kilpatrick (2003). S. 75-76 betont, dass die Biographie des Mughira aufgrund ihrer Detailfreude besonders hervorsticht.

55 Siehe. die Hinweise auf weitere Quellen in Lammens (2015); der Historiker al-Tabari berichtet darüber in seinem Eintrag zum Jahr 17, allerdings unter Berufung auf andere Überlieferer und mit anderen Versionen. S. al-Tabarī (1879-1901). Ta’rikh al-mulük. Hg. Michael Jan de Goeje. Leiden: Brill. S. 2529-2531.

56 Im Koran wird die Auspeitschung vorgeschrieben. Als juristischer Usus, mit Berufung auf die Prophetentradition, setzte sich aber die Steinigung durch; s. Peters (2015).

$57 \mathrm{Zu}$ diesem Erfordernis des islamischen Rechts s. Anm. 50.

58 Alle anderen Episoden in der Biographie des Mughira, die zuvor al-Iṣfahānī erzählt, tragen zu dieser Charakterisierung weiter bei.

59 al-Ișfāhāni (1927-1961), Bd. XVI. S. 97.

60 Ebd., S. 96-98. 
einen Sexualakt beobachtet zu haben, den er detailliert beschreibt, was noch eine zusätzliche, schlüpfrige Pointe bedeutet (z.B. „ich sah das Wackeln der Hoden" $\left.{ }^{61}\right)$, sein Zeugnis aber dennoch nicht den formalrechtlichen Erfordernissen genügt: Der Zeuge kann nämlich nicht den eigentlichen Koitus einwandfrei bezeugen, denn er hätte bezeugen sollen, dass der Penis „wie ein Kohlstift in seinem Behälter auf und ab geht" ${ }^{62}$ Aus dieser Version der Geschichte in dem „Buch der Lieder“ gewinnt der Leser den Eindruck, dass Mughira zwar einerseits schuldig war (schließlich befand er sich im Haus der Frau und hatte in Gegenwart von Zeugen offensichtlich Geschlechtsverkehr mit ihr), aber dennoch sein Vergehen nicht geahndet wurde, weil sich Mughira mit Hilfe seiner List aus der schwierigen Lage befreien konnte.

Interessant sind die zahlreichen gesellschaftskritischen Implikationen der Geschichte, die aus dem Bașra der vorbildhaften frühislamischen Zeit einen korrupten Ort der Sünde machen. So ist es generell bemerkenswert, dass al-Iṣfahāni den Gouverneur Mughïa als Schlitzohr porträtiert, obwohl es sich um einen angesehenen Prophetengefährten handelt. ${ }^{63}$ In eine ähnliche Richtung weist auch die Szene, in der Mughira seine Position bei den Prophetengefährten geltend macht, um diese tränenreich dazu zu bewegen, ihn zu unterstützen und auch Druck auf den Zeugen Ziyād auszuüben. ${ }^{64}$ Somit erscheint nicht nur Mughira als weinerlicher Manipulator, sondern die gesamte Gruppe der Prophetengefährten als manipulierbar und korrupt. Auch der Kalif 'Umar spielt eine sehr ambivalente Rolle, da er das Leben des Mughira trotz dessen Schuld retten möchte und sich freut, als dieser nicht verurteilt wird. ${ }^{65}$ Schließlich erweist sich auch der Nachfolger des Mughira als korrupt, er nimmt nämlich als Dank für seine „kooperative“ Haltung eine schöne Sklavin von dem Mughira als Geschenk an. ${ }^{66}$ Ein weiteres Thema ist die Machtlosigkeit des Gesetzes, das diesen Missstand offensichtlich nicht bekämpfen konnte, da es viel zu formalistisch verstanden wurde. Hier findet sich also auch eine implizite Kritik an der Wirksamkeit des fiqh (islamisches Recht) als moralisch regelnde Kraft der Gesellschaft.

Dies alles deutet auf eine Literarisierung des Erzählstoffes und auch auf eine sehr ironische Herangehensweise des al-Ișfahānī hin, der eine historische Erzählung über den Liedtextverfasser Mughira in eine unterhaltsame gesellschaftskritisch-satirische Fabel über Macht und Korruption verwandelt, wodurch er die In-

61 Ebd., S. 98.

62 Siehe Anm. 50.

63 Die Muslime erster Stunde, die zu Lebzeiten des Propheten zum Islam konvertierten, werden „Prophetengefährten“ ( $(a h a \bar{a} b a)$ genannt und dienen bis heute nach dem Propheten als Richtschnur für moralisch vorbildliches Verhalten bei den Sunniten. Miklos Muranyi (2015) „Șahāba“. Encyclopaedia of Islam. Second Edition. Hgg. P. Bearman u.a. Brill Online. al-Iṣfāhāni (1927-1961), Bd. XVI. S. 97.

65 Ebd., S. 98-99.

66 Ebd., Bd. XVI. S. 96. 
tegrität der vermeintlich vorbildhaften Frühzeit des Islam und somit eines in seiner Zeit anerkannten Wertes in Frage stellt. ${ }^{67}$ Dies verweist auf eine typische Fiktionalisierung des vorgegebenen Stoffes (die Biographie des Mughira) bzw. auf eine funktionale Fiktionalisierung mit moralischen Implikationen.

Die wiederholte und detaillierte Nennung von historischen Personen, Ortsnamen und anderen Details deutet jedoch auf Faktualität. Wichtige Indizien sind ferner die akribische und korrekte Verwendung des isnād, die den Erzählfluss mehrfach unterbricht; wie auch das Zitieren von ergänzenden und abweichenden Parallelversionen, die an die Praxis von Historikern wie al-Tabarī erinnert. Al-Iṣfahānì verwendet hier die beiden Authentifizierungsstrategien, die wir bei Historikern desselben Zeithorizonts finden. Er wendet also Konventionen an, die Faktizität evozieren.

\section{Zusammenfassung}

Die schöngeistige MHAL oder $a d a b$ ist ein gutes Beispiel für eine nicht europäische ältere Literatur, deren narrativer Status schwer zu bestimmen ist. Die hier untersuchten Passagen wiesen einerseits einige Merkmale auf, die auf einen faktualen Status hinweisen und Wirklichkeitsnähe evozieren, dazu zählt die Kontrastierung von Paralleltexten, die Verwendung historischer Persönlichkeiten und topographischer Gegebenheiten sowie ein realistischer Stil. Es überwogen allerdings Signale, die auf eine funktionale Fiktionalität hinwiesen; hierzu zählt eine starke Literarisierung des Erzählstoffes, der politisch-moralische Gehalt wie auch die Rhetorizität der Erzählungen. Hier finden sich viele Parallelen zur europäischen mittelalterlichen Literatur.

Ein kulturspezifisches Merkmal ist hingegen der sogenannte isnād (Autoritätenkette), der in seinem Originalkontext als die zentrale Authentifizierungskonvention der Prophetentradition und Geschichtsschreibung fungierte. Wir dürfen also davon ausgehen, dass der isnäd im Rahmen der arabisch-islamischen Kultur konventionell auf faktuale Textgruppen verweist. Seine Ausbreitung, Verwendung und Funktion im Kontext von adab-Sammlungen ist uneinheitlich und noch wenig erforscht. Während er im „Halsband“ (Text 1) ausdrücklich ausgelassen wurde, wird er im „Buch der Lieder“ (Text 2) in voller Ausführlichkeit und sehr akkurat verwendet. Dies ist ein signifikanter Unterschied, der möglicherweise sowohl auf einen unterschiedlichen narratologischen wie auch epistemischen Status beider Texte hinweist. Die Gültigkeit der Texteinheiten im „Halsband“ wäre damit universell und kontextunabhängig, während sie im „Buch der Lieder“ eher historisch wäre. Zum Gebrauch des isnād außerhalb der Prophetentradition

67 Diese kritische Haltung könnte damit zusammenhängen, dass al-Ișfahānī Schiit war und somit die in der Sunna verehrten Werte, wie z.B. der besondere Status der Prophetengefährten, kritisch sah. 
und der Geschichte liegen allerdings leider noch keine signifikanten Vorarbeiten vor; hier sind auf jeden Fall vertiefende Studien erforderlich.

\section{Literatur}

Abū al-Farağ al-Iṣfāhānī (1927-1961) Kitāb al-Ag̀̄annì. Kairo: Dār al-Kutub.

Ashtiany, Julia u.a., Hg. (1990) The Cambridge History of Arabic Literature. 'Abbasid Belles-Lettres. Cambridge: Cambridge University Press.

Beeston, A. F. (1983) „The role of parallelism in Arabic prose“. The Cambridge History of Arabic Literature. Arabic Literature to the end of the Umayyad period. S. $180-185$

Bray, Julia (2005) „'Abbasid Myth and the Human Act: Ibn 'Abd Rabbih and Others". On Fiction and Adab in Medieval Arabic Literature. Hg. Philip Kennedy. Leiden: Brill. S.1-54.

Brown, Jonathan A. C. (2009) Hadith. Oxford: OneWorld. S. 67-122

Fähndrich, Hartmut (1990) „Der Begriff ,adab‘ und sein literarischer Niederschlag". Neues Handbuch für Literaturwissenschaft. Bd. 5: Orientalisches Mittelalter. Hg. Wolfhart Heinrichs. Wiesbaden: AULA Verlag S. 326-345.

Fierro, Isabel (1989) „The introduction of hadith in al-Andalus“. Der Islam 66, 1. S. 68-93.

Hämeen-Anttila, Jaakko (2015) „Adab a) Arabic, early developments“. Encyclopaedia of Islam, THREE. Hgg. Kate Fleet u.a. Brill Online.

Hasson, I. (2015), „Ziyād b. Abihi“. Encyclopaedia of Islam, Second Edition. Hgg. P. Bearman u.a. Brill Online.

Houtsma, M.Th./Pellat, Ch. (2015) „Abū Bakra“. Encyclopaedia of Islam. Second Edition. Hgg. P. Bearman u.a. Brill Online.

Hoyland, Robert G. (2006) „History, Fiction and authorship in the first centuries of Islam". Writing and Representation in Medieval Islam. Hg. Julia Bray. Routledge: London. S. 16-46.

Ibn 'Abd Rabbih (1990) al-`Iqd al-farìd. Hgg. Amin/al-AbyārīTudmirī. Beirut: Dar al-Kutub al-Tlmiya.

Kennedy, Philip (2005) „Preface“. On Fiction and Adab in Medieval Arabic Literature. Hg. Philip Kennedy. Leiden: Brill. S. xi-xxii.

Khalidi, Tarif (1994) Arabic Historical Thought in the Classical Period. Cambridge: Cambridge University Press.

Kilpatrick, Hilary (2003) Making the Great Book of Songs. London: Routledge.

Lammens, Henri (2015) „al-Mughira b. Shu'ba“. Encyclopaedia of Islam. Second Edition. Hgg.. P. Bearman u.a. Brill Online.

Lecker, Michael (2015) „Thakif“. Encyclopaedia of Islam. Second Edition. Hgg. P. Bearman u.a. Brill Online. 
Leder, Stefan (1998) „Conventions of Fictional Narration in Learned Literature“. Story-telling in the framework of non-fictional Arabic literature. Hg. Stefan Leder. Wiesbaden: Harrassowitz. S. 34-60.

Leder, Stefan/Hilary Kilpatrick (1992) „Classical Arabic Prose Literature. A Researcher's Sketch Map“. Journal of Arabic Literature 23, 1. S. 2-26.

Menninghof, Burckhard (2007) „Literatur“, Metzler Lexikon Literatur. Hgg. Dieter Burdorf/ Christoph Fasbender/Burckhard Moennighoff. Stuttgart: Metzler. S. $445 a-445 b$.

Moscati, Sabatino (1950) „Lemassacre des Umayyades“. Archiv orientální 28, 4. S. 88-115.

Müller, Jan-Dirk (2004) „Literarische und andere Spiele. Zum Fiktionalitätsproblem in vormoderner Literatur." Poetica 36. S. 281-312.

Muranyi, Miklos (2015) „Șahāāba“. Encyclopaedia of Islam. Second Edition. Hgg. P. Bearman u.a. Brill Online.

Orfali, Bilal (2012) „A Sketch Map of Arabic Poetry Anthologies up to the Fall of Baghdad“. Journal of Arabic Literature 43. S. 29-69.

Peters, R. (2015) „Zinā'`“. Encyclopaedia of Islam. Second Edition. Hgg. P. Bearman u.a. Brill Online.

Rotter, Gernot (2004) Und der Kalif beschenkte ibn reichlich. Auszüge aus dem „Buch der Lieder". Lenningen: Erdmann.

Safran, Janina (2000) The Second Umayyad Caliphate: The Articulation of Caliphal Legitimacy in al-Andalus. Cambridge: Harvard University Center for Middle Eastern Studies.

Scheiner, Jens (2016) „Der Hadit“. Islam. Hg. Rainer Brunner. Stuttgart: Kohlhammer. S.110-131

Țabarī (1879-1901) Ta’rīkh al-mulük. Hg. Michael Jan de Goeje. Leiden: Brill.

Toral-Niehoff, Isabel (2010) „,Sei seine Dienerin, dann wird er dein Diener sein! Auf der Suche nach der idealen Ehefrau: Ibn 'Abd Rabbihi und sein Buch über die Frauen". Didaktisches Erzäblen. Spielarten literarischer Belebrung in Orient und Okzident. Hgg. Regula Forster/Romy Günthart. Berlin: Peter Lang S. 255-275.

--- (2015a) „,Fact and Fiction' in der mittelalterlichen arabischen Literatur. Anmerkungen zu einer Debatte“. Faktuales und fiktionales Erzäblen. Interdisziplinäre Perspektiven. Hgg. Monika Fludernik/Nicole Falkenhayner/Julia Steiner. Würzburg: Ergon. S. 59-75.

--- (2015b) „The ,Book of the Pearl on the Ruler' in the Unique Necklace by Ibn 'Abdrabbih: Preliminary Remarks". New Approaches to the History of Political Thought: Mirrors of Princes Reconsidered. Hgg. Neguin Yavari/Regula Forster. Cambridge: Harvard University Press. S. 134-151.

Toral-Niehoff, Isabel (2015c) „History in Adab Context: ,The Book on Caliphal Histories' by Ibn 'Abd Rabbih (246/860-328/940)“. Journal of Abbasid Studies 2. S. 61-85. 
Van Laak, Lothar (2007) „Fiktionalität“. Metzler Lexikon Literatur. Hgg. Dieter Burdorf/Christoph Fasbender/Burckhard Moennighoff. Stuttgart: Metzler. S. 240a-242a.

Werkmeister, Walter (1983) Quellenuntersuchungen zum Kitäb al-`Iqd al-Farìd des Andalusiers Ibn' Abdrabbih (246/840-328/940). Berlin: Schwarz.

Young, M. J. L./J. D. Latham/R. B. Serjeant, Hgg. (1990) The Cambridge History of Arabic Literature. Religion, learning and science in the 'Abbasid period. Cambridge: Cambridge University Press. 